\title{
Eisen-, Kupfer- und Zinkanalysen unter Anwendung der Atom-Absorptions-Spektralphotometrie
}

\author{
Von G. HeINEMANN \\ Aus dem Institut für Klinische Chemie und Klinische Biochemie (Direktor: Prof. Dr. H.-J. Dulce) \\ am Klinikum Steglitz der Freien Universität Berlin
}

(Eingegangen am 7. Februar/31. Juli 1972)

\begin{abstract}
Es werden einfache Bestimmungen für Eisen im Plasma sowie Kupfer und Zink im Serum ohne Enteiweißung beschrieben. Auf Schwierigkeiten und Fehlermöglichkeiten - insbesondere die Hämolyse bei der Eisenbestimmung - wird hingewiesen. Zur Kennzeichnung des physiologischen Bereichs von Eisen im Plasma, Kupfer und Zink im Serum werden 95\%-Normbereiche angegeben, die wir aus den Plasma- bzw. Serumwerten von 136 Frauen und 104 Männern errechneten. Die Frauen nahmen keine Ovulationshemmer. Die angeführte Methode erfordert geringen Arbeitsaufwand, ist zeitsparend und im Hinblick auf die Zuverlässigkeitskriterien als Methode für Serienbestimmungen geeignet. Die Methode ist jeweils für die Elemente Eisen, Kupfer und Zink spezifisch.
\end{abstract}

\section{Iron, copper, and zinc analyses by atomic absorption spectrophotometry}

Simple determinations for iron in plasma and copper and zinc in serum, which do not require deproteinization, are described. Difficulties and possibile errors - especially hemolysis in the iron determination - are pointed out. To establish the physiological ranges of iron in plasma, and copper and zinc in serum, we determined the $95 \%$ norm range from the plasma or serum values of 136 women and 104 men. The women were not taking ovulation inhibitors. The method presented here requires low labor expenditures, saves time, and, with respect to reliability, is suitable for series determinations. The method is specific for the elements iron, copper and zinc.

Die Anwendung der Atom-Absorptions-Spektralphotometrie zum Nachweis von Metallen, Alkalien und Erdalkalien hat aufgrund ihrer schnellen und präzisen Technik einen festen Platz in der Analytik eingenommen.

Die Probenvorbereitung zur Bestimmung von Eisen im Plasma, Kupfer und Zink im Serum erfordert allerdings mehrfache Pipettierungen, die für routinemäßige Analysen eine wesentliche Fehlerqueile bedeuten. Das zu bestimmende Element wird mit Hilfe eines Komplexbildners in ein zugesetztes organisches Lösungsmittel überführt und erfährt damit eine Anreicherung. Zweitens wird eine Enteiweißung empfohlen, um das Verstopfen. des Brennerkopfes zu verhüten.

Es hat nicht an Versuchen gefehlt, die Probenvorbereitung abzuwandeln und auf die Enteiweißung zu verzichten. Einen ersten Hinweis zur direkten Messung von Eisen, Kupfer und Zink im Serum gaben SpRAGUE und Stavin (1), die Proteinlösungen als Basis der Eichlösungen empfahlen. Später berichteten HACKLEY et al. (2), Ringhardtz und Welz (3) und Parker et al. (4) über direkte Verfahren unter Verwenden von Dextranen bei der Herstellung der Eichlösungen. Andere Autoren wie Fuwa et al. (5) und Schnidr (6) wiederum verdünnten lediglich das Serum und maßen gegen wäßrige Eichlösungen. Dịe Normbereiche, die mit der Atom-Absorptions-Spektralphotometrie nach verschiedenen möglichen Methoden ermittelt wurden, differieren.

Wir stellten uns die Aufgabe, für die routinemäßige Bestimmung von Eisen im Plasma, Kupfer und Zink im Serum Verfahren zu entwickeln, die wenig Plasma oder Serum verbrauchen, eine direkte Messung gestatten, lineare Eichkurven ergeben und schnell durchzuführen sind.

Wir ermittelten Normbereiche für Eisen im Plasma sowie für Kupfer und Zink im Serum bei Frauen, die keine Ovulationshemmer einnahmen, und bei Männern. ". Wir prüften Zuverlässigkeit und Korrelation zụ anderen Methoden.

\section{Methodik und Probanden}

\section{Apparatur}

Wir benutzten das Atom-Absorptions-Spektralphotometer Modell 303 der Firma Perkin-Elmer mit angeschlossenem DCR-1 (Digital-Concentration-Readout). Als Brenner verwendeten wir aufgrund seiner Vorzüge den Boling-Dreischlitzbrenner, als Hintergrundstrahler die elementspezifischen Intensitron-Lampen von Perkin-Elmer. Die Geräte wurden mindestens $20 \mathrm{~min}$ vor $\mathrm{MeB}$ beginn eingeschaltet, die Flamme etwa $10 \mathrm{~min}$ vor Meßbeginn gezündet. Die Geräteeinstellung für das Atom-AbsorptionsSpektralphotometer ist in Tabelle 1 aufgeführt.

\section{Ansaugvolumen}

Das Ansaugvolumen wurde festgelegt durch Verwenden eines englumigen PVC-Schlauches von $14,5 \mathrm{~cm}$ Länge, der eine Zerstäubung von etwa $2,3 \mathrm{ml}$ Wasser/min gewährleistete. Eichlösungen und verdünntes Serum sollten die gleiche Viskosität aufweisen. Wie Vorversuche ergaben, erwies sich neo-Plasmagel zur Herstellung der Eichlösungen als geeignet.

\section{Reagenzien}

Ammoniumeisen (Il)-sulfat p. a. (Merck) $\left(\mathrm{NH}_{4}\right)_{2} \mathrm{Fe}\left(\mathrm{SO}_{4}\right)_{2} \cdot 6 \mathrm{H}_{2} \mathrm{O}$. Kupfer (II)-sulfat p. a. (Merck) $\mathrm{CuSO}_{4} \cdot 5 \mathrm{H}_{2} \mathrm{O}$.

Zinksulfat p. a. (Merck) $\mathrm{ZnSO}_{4} \cdot 7 \mathrm{H}_{2} \mathrm{O}$.

Lanthanoxid puriss. (Roth) $\mathrm{La}_{2} \mathrm{O}_{3}$.

Konz. Salzsäure p. a. 37 proz. (Merck). 
Tab. 1

Geräteinstellung für das Atom-Absorptions-Spektralphotometer zur Bestimmung von Eisen im Plasma, Kupfer und Zink im Serum

\begin{tabular}{|c|c|c|c|}
\hline & \multicolumn{3}{|c|}{ Bestimmung von:- } \\
\hline & $\mathrm{Fe}$ & $\mathrm{Cu}$ & $\mathrm{Zn}$ \\
\hline Wellenlänge [nm] & 248,4 & 325,0 & $214 ; 0$ \\
\hline Spaltbreite & 3 & 4 & 5 \\
\hline Lampenstrom [mA] & 30 & 15 & 15 \\
\hline Brenner & \multicolumn{3}{|c|}{$\begin{array}{l}\text { Boling- } \\
\text { Brenner }\end{array}$} \\
\hline Brennerhöhe & \multicolumn{3}{|c|}{$\begin{array}{c}\text { Einstellung nach Absorptions- } \\
\text { maximum }\end{array}$} \\
\hline Skalendehnung & keine & keine & keine \\
\hline Dämpfung & 3 & 3 & 3 \\
\hline Flamme & & $\begin{array}{c}\text { brennga } \\
\text { arm }\end{array}$ & \\
\hline Brenngas & & $\begin{array}{r}\text { Acetyle } \\
\text { etwa } 91 / 1\end{array}$ & \\
\hline Oxidans & & $\begin{array}{r}\text { Preßluf } \\
\text { etwa } 91 / x\end{array}$ & \\
\hline
\end{tabular}

neo-Plasmagel $30 \mathrm{~g} / 1$ (B. Braun, Melsungen) Zusammensetzung: Modifizierte flüssige Gelatine $30 \mathrm{~g} / \mathrm{l}$, Natrium $142 \mathrm{mmol} / \mathrm{l}$, Calcium $1,4 \mathrm{mmol} / \mathrm{l}$, Chlorid $109 \mathrm{mmol} / \mathrm{l}$.

Für die vergleichenden Eisen- und Kupferbestimmungen benutzten wir den Merckotest "Eisen“ (Merck) und den Farbtest "Kupfer" (C. F. Boehringer) im Mikrolitermaßstab.

Seronorm (Nyegaard \& Co, Oșlo).

\section{Losungen}

1. Lanthanlösung $50 \mathrm{~g} / 1: 58,64 \mathrm{~g} \mathrm{La}_{2} \mathrm{O}_{3}+169,0 \mathrm{ml}$ konz. Salzsäure ad 1 Liter tridest. Wasser.

2. Stammlösung $1 \mathrm{mmol} / \mathrm{l}$ für Ammoniumeisen(II)-sulfat, Kupfer (II)-sulfat und Zinksulfat: Eingewogene Menge $+10 \mathrm{ml}$ konz. Salzsäure ad 1 Liter Lösung 3.

3. neo-Plasmagel-Lanthanlösung: $100 \mathrm{ml}$ Lösung $1+400 \mathrm{ml}$ tridest. Wasser ad 1 Liter neo-Plasmagel.

4. Eichlösungen für $\mathrm{Fe}, \mathrm{Cu}$ und $\mathrm{Zn}: 1,2,4,6$ und $8 \mathrm{ml}$ der Lösung 2 ad $100 \mathrm{ml}$ Lösung $3=10,20,40,60$ und $80 \mu \mathrm{mol} / 1 \mathrm{Fe}, \mathrm{Cu}$ und $\mathrm{Zn}_{\mathrm{n}}$.

5. Lanthanlösung $10 \mathrm{~g} / \mathrm{l}: 200 \mathrm{ml}$ Lösung 1 ad 1 Liter tridest. Wasser.

\section{Probenvorbereitung}

$0,5 \mathrm{ml}$ der Lösung 5 werden zu $0,5 \mathrm{ml}$ hämolysefreiem Plasma für die Eisenbestimmung und zu je $0,5 \mathrm{ml}$ hämolysefreiem Serum für die Kupfer- und Zinkanalysen pipettiert. Die Probenmengen sind ausreichend für mindestens 10 Einzelwerte, sofern am DCR-1 der Schalter Average auf „repeat“ gestellt wird.

\section{Probanden}

Die physiologischen Bereiche für Eisen im Plasma, Kupfer und Zink im Serum wurden bei 136 Frauen und 104 Männern im Alter $z$ wischen 20 und 60 Jahren ermittelt, bei denen es sich um gesunde Personen verschiedener Berufsgruppen handelte. Das Blut wurde den nüchternen Personen zwischen 8.00 und 9.00 Uhr entnommen.

\section{Ergebnisse}

\section{Linearität}

Linearität ergab. sich für die' Eisen-, Kupfer- und Zinkeichlösungen im Bereich von $0-80 \mu \mathrm{mol} / 1$ (Abb. 1). Höhere Konzentrationen führten zu einer Kurvendepression. Die Extinktionswerte der Eichlösungen auf Abbildung 1 entsprechen einem Mittelwert aus 10 Einzelwerten, wobei die Streuung um den Mittelwert sehr gering war (Tab. 2).

\section{Zuverlässigkeit}

Das zur Herstellung der Eichlösungen verwendete neo-Plasmagel ist eine wäßrige, elektrolythaltige Gelatinelösung, deren Gelatineanteil aus tierischem Knochengewebe gewonnen wird. Wir haben daher den Eisen-, Kupfer- und Zinkgehalt im neo-Plasmagel atomabsorptionsphotometrisch bestimmt und fanden $1,2 \mathrm{nmol} / \mathrm{l}$ Eisen, $0,96 \mathrm{nmol} / \mathrm{l} \mathrm{Kupfer}$ und $0,72 \mathrm{nmol} / \mathrm{l}$ Zink. $1 \mathrm{~g}$ Lyophilișat von neo-Plasmagel wurde im Plantintiegel verascht, in $4 \mathrm{ml} \mathrm{HCl}, 0,1 \mathrm{~mol} / \mathrm{l}$, aufgenommen und gegen wäßrige Eichlösungen gemessen. $\mathrm{Da}$ für die Eichlösungen ein 1:2 verdünntes neo-Plasmàgel benutzt wird, liègen die Eisen-, Kupferund Zinkkonzentrationen darin um den Faktor $2-6 \cdot 10^{2}$ unter der Nachweisgrenze des Atom-Absorptions-Spektrometers, sind also zu vernachlässigen.

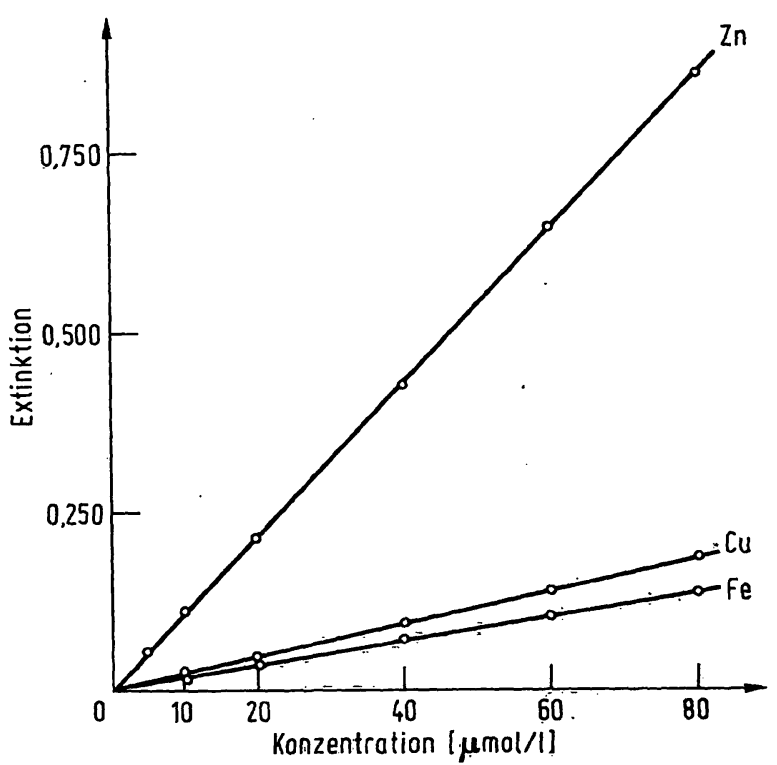

Abb. 1

Linearität der Eichgeraden bis $80 \mu \mathrm{mol} / 1$ bei minimaler Standardabweíchung der Einzèlwerte (s. Táb. 2)

Tab. 2

Mittelwert $(\overline{\mathbf{x}})$, Standardabweichung (s) und Variationskoeffizient (\%) von je 10 Extinktionen für Eisen, Kupfer und Zink

\begin{tabular}{|c|c|c|c|c|c|c|c|c|c|}
\hline $\begin{array}{c}\text { Eichpunkte } \\
\mu \mathrm{mol} / 1\end{array}$ & $\overline{\mathbf{x}}$ & $\begin{array}{c}\text { Eisen } \\
\pm \mathrm{s}\end{array}$ & VK & $\overline{\mathbf{x}}$ & $\begin{array}{c}\text { Kupfer } \\
\pm s\end{array}$ & VK & $\overline{\mathbf{x}}$ & $\begin{array}{c}\text { Zink } \\
\text { 土s }\end{array}$ & VK \\
\hline 10 & 0,0169 & 0,00028 & 1,65 & 0,0234 & 0,00040 & 1,72 & 0,1068 & 0,00062 & 0,58 \\
\hline 20 & 0,0345 & 0,00058 & 1,68 & 0,0472 & 0,00034 & 0,73 & 0,2170 & 0,00084 & 0,39 \\
\hline 40 & 0,0712 & 0,00074 & 1,04 & 0,0972 & 0,00047 & 0,48 & 0,4339 & 0,00133 & 0,30 \\
\hline 60 & 0,1051 & 0,00090 & 0,86 & 0,1427 & 0,00080 & 0,56 & 0,6636 & 0,00208 & 0,31 \\
\hline 80 & 0,1393 & 0,00095 & 0,68 & 0,1946 & 0,00156 & 0,80 & 0,8605 & 0,00193 & 0,22 \\
\hline
\end{tabular}




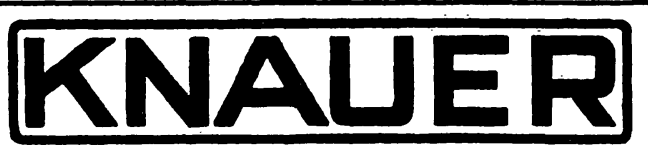

ELEKTRON ISCHES HALBMIKRO - OSMOMETER

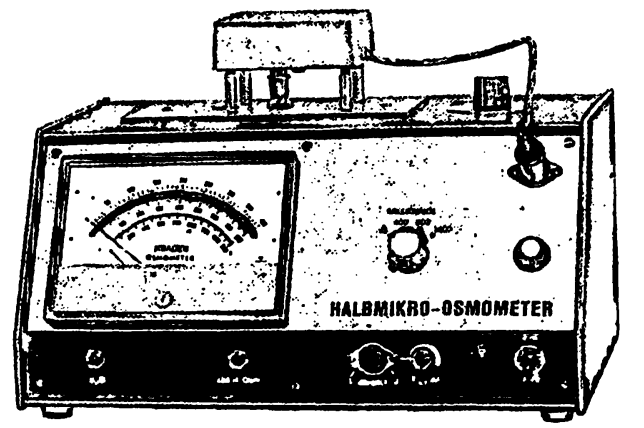

zur direkten Bestimmung der Osmolalität aller Körperflüssigkeiten wie Blut, Serum, Urin, Liquor durch Gefrierpunktmessung. Kontrolle der Nierenfunktion.

- Probevolumen nur $0,15 \mathrm{ml}$ oder $0,05 \mathrm{ml}$

- Meßgenavigkeit 1-2 Milliosmol/kg bzw. 1\%

- Preis DM 3600, - + MWSt

NEU: Vollautomatisches Osmometer OSMOPRINT für 20 Proben, mit digitaler Anzeige und eingebautem Drucker zu $8600,-$ - MWSt. Geräte zur GEL-CHROMATOGRAPHIE

Weitere Spezialität: Komplettes System zur Molekulargewichtsbestimmung zwischen 100 und 1000000 durch Kryoskopie, Dampfdruck-Osmometrie und Membran-Osmometrie.

KG Dr.-Ing. Herbert Knaver \& Co. GmbH,

1 Berlin 37, Holstweg 18, Tel. 848705

Pharmazie-Ausstellung, Düsseldorf 12.-15.10.72 Halle 3, Stand 3084

\section{Walter de Gruyter Berlin. New York}

\section{Küster - Thiel - Fischbeck Logarithmische Rechentafeln}

für Chemiker, Pharmazeuten, Mediziner und Physiker

Begründet von F.W. Küster, fortgeführt von A. Thiel, neubearbeitet von $K$. Fischbeck

101., verbesserte und erweiterte Auflage. Oktav. XVI, 313 Seiten, 1 Mantissentafel. Mit Griffregister. 1972. Gebunden DM 26, - ISBN 3110039737

(Arbeitsmethoden der modernen Naturwissenschaften)

\section{Szintillations-Zählfläschchen}

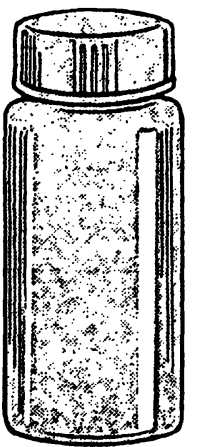

aus ND-Polyäthylen und kaliumarmem Glas

\section{Chemikalien}

für die Flüssigszintillation liefern wir

schnell und preiswert

Fordern Sie bitte unsere

neve Preisliste an

\section{Werner Zinsser \\ Scintillators}

6000 FRANKFURT 1, NORDENDSTR. 20, ABT. 17

\section{Laborgeräte aus PTFE \\ (Hostaflon(R) $^{\left(\text {Teflon }^{(R)}\right)}$ \\ Autoklaven und Filtrationszellen \\ Membranen und Filterplatten}
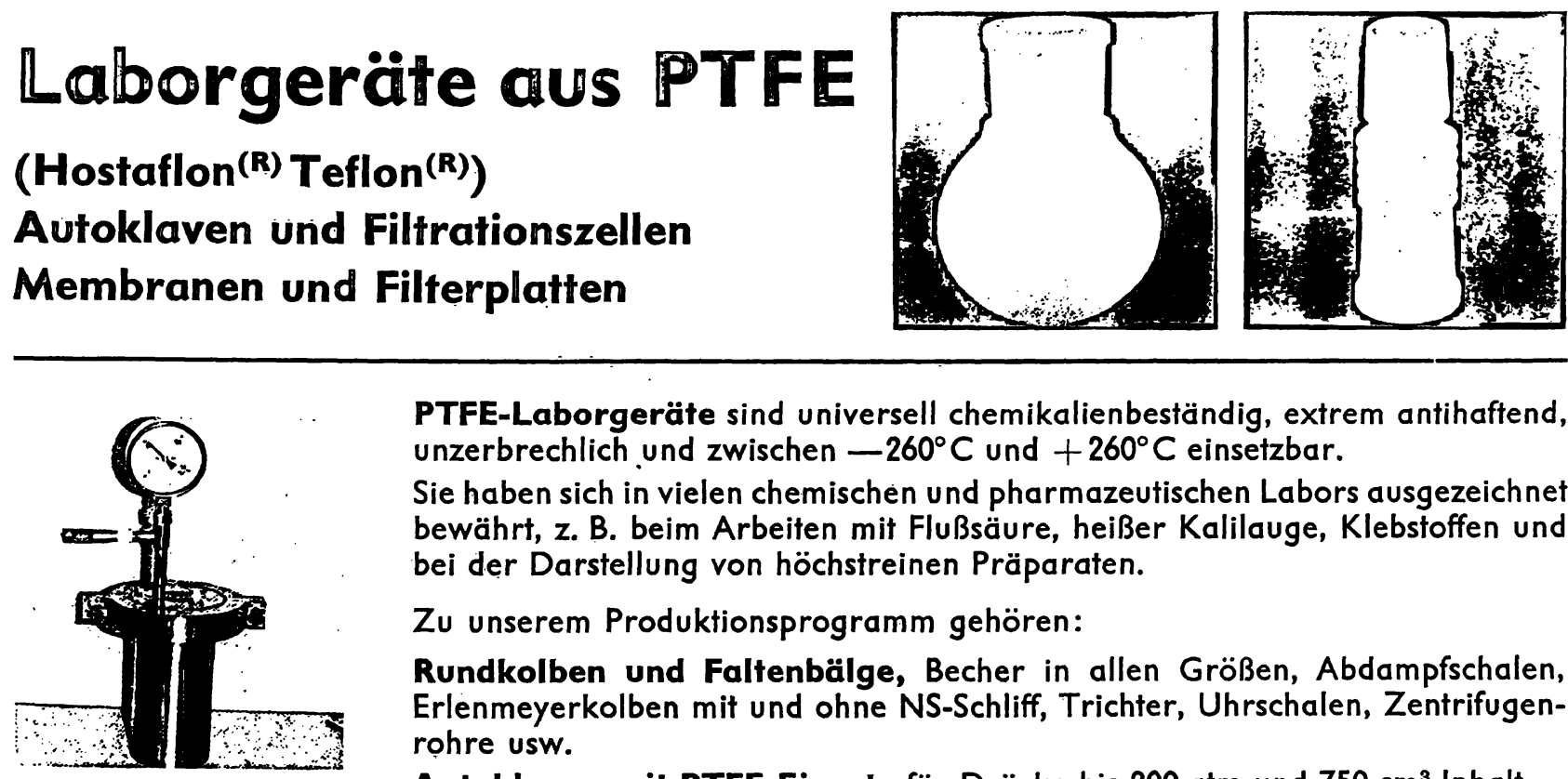

PTFE-Laborgeräte sind universell chemikalienbeständig, extrem antihaftend, unzerbrechlich und zwischen $-260^{\circ} \mathrm{C}$ und $+260^{\circ} \mathrm{C}$ einsetzbar.

Sie haben sich in vielen chemischen und pharmazeutischen Labors ausgezeichnet bewährt, z. B. beim Arbeiten mit Flußsäure, heißer Kalilauge, Klebstoffen und bei der Darstellung von höchstreinen Präparaten.

Zu unserem Produktionsprogramm gehören:

Rundkolben und Faltenbälge, Becher in allen Größen, Abdampfschalen, Erlenmeyerkolben mit und ohne NS-Schliff, Trichter, Uhrschalen, Zentrifugenrohre usw.

Autoklaven mit PTFE-Einsatz für Drücke bis $200 \mathrm{~atm}$ und $750 \mathrm{~cm}^{3}$ Inhalt. Filtrationszellen aus Kunststoff oder Edelstahl für hydrostatische Drücke bis zu $150 \mathrm{~atm}$.

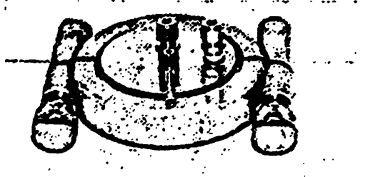

Membranen und Filterplatten mit verschiedenen Porengrößen zwischen 0,05 und $50 \mu$ zur Reinigung. Trennung und Konzentrierung von Mischungen und makromolekularen Lösungen.

Nach Ihren speziellen Anforderungen stellen wir Sonderapparate her. 
Sauer, Dr. Friedhelm

\section{Einführendes Lehrbuch der Biochemie für Mediziner} und Biologen

In der Reihe "Manumed"

VIII, 444 Seiten, Schreibsatz, 719 Formeln und Abbildungen; kt. DM 20,-

Dieses kurzgefaßte und preiswerte Lehrbuch wendet sich an Studierende, die mit dem Grundwissen der Chemie bereits vertraut sind und hier nun der Biochemie, als Basiswissenschaft dargestellt, begegnen.

Sauer, Dr. Friedhelm

Aufgabensammlungen

zur Vorbereitung auf das Physikum und zum Physiologisch-Chemischen Praktikum. Mit Antworten.

In der Reihe "Examina medica"

76 Seiten, Schreibsatz, kt. DM 8,-

Berg, Oberpräparator Georg

Histologische Labortechnik

1972, 174 Seiten mit 8 farbigen und 3 Schwarzweiß-Abbildungen

Plastik DM 28,- Kt. DM 25,-

Das vom erfahrenen Praktiker verfaßte Buch ist das ideale Lernmittel für alle einschlägigen Fachschulen, denn es schildert alle anfallenden Arbeiten von der Bereitstellung der Reagenzien bis zu neuzeitlichen Färbemethoden und dem Schneiden am Mikrotom.

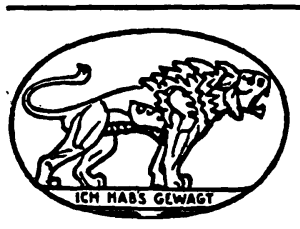

\section{J.F.LEHMANNS VERLAG MÜNCHEN}

\section{BIOCHIMIE}

Edité par la Société de Chimie Biologique

tel est le titre

sous lequel paraîtra à partir de 1971

le „BULLETIN DE LA SOCIÉTÉ DE CHIMIE BIOLOGIQUE““

\section{SECRÉTARIAT}

de la Société de Chimie Biologique

J. P. EBEL, Secrétaire Général (Relations Extérieures)

R. PERLES, Secrétaire Général

\section{REDACTION}

F. GROS, Secrétaire scientifique

F. PERCHERON, Secrétaire à la Publication J. NUNEZ, Secrétaire à l'Information

Y. RAOUL, Secrétaire à l'Edition

SECRETARIAT et REDACTION: 4 Avenue de l'Observatoire, PARIS $6^{\circ}$

12 FASCICULES

ABONNEMENTS: FRANCE et ZONE FRANC: $150 \mathrm{ffrcs}$ - BELGIQUE: 1.687,- frcs - AUTRES PAYS: 186, - ffrcs MASSON et Cie, Editeurs - 120 Boulevard St Germain - PARIS 6éme 


\section{Ricbtigkeit}

Zur Prüfung der Richtigkeit der Eisen-, Kupfer- und Zinkbestimmung wurde die Additionsmethode angewandt. Im ersten Teil dieser Versuche stellten wir das DCR-1 auf "Absorbance" (= Extinktion) und ermittelten aus den Extinktionswerten die Konzentrationen mit Hilfe der Eichkurve nach der Formel

$$
C_{p}=\frac{E_{p} \cdot C_{s}}{E_{s}} \cdot F
$$

$C_{p}=$ Konzentration im Serum oder Plasma, $C_{s}=$ Konzentration des Eichwertes, $E_{p}=$ Extinktion der Probe, $\mathrm{E}_{\mathrm{s}}=$ Extinktion des Eichwertes, $\mathrm{F}=$ Verdünnungsfaktor.

Arbeitsgang (Additionsmethode): $\mathrm{Zu}$ je $4,5 \mathrm{ml}$ eines gepoolten Humanserums (Gehalt an Eisen: $21,4 \mu \mathrm{mol} / 1$, Kupfer: $20,0 \mu \mathrm{mol} / 1$ und Zink: $13,0 \mu \mathrm{mol} / \mathrm{l}$ ) wurden $0,5 \mathrm{ml}$ einer wäßrigen Lösung der Konzentration 50 , 100, 150 und $300 \mu \mathrm{mol} / \mathrm{l}$ Eisen bzw. Kupfer bzw. Zink gegeben. Das Ergebnis der Wiederfindung zeigt Tabelle 3.

Die Wiederfindung im Bereich unterhalb von $10 \mu \mathrm{mol} / \mathrm{l}$ wurde geprüft für 2,4 und $5 \mu \mathrm{mol} / 1$ Eisen sowie für 2 und $5 \mu \mathrm{mol} / 1$ Kupfer und Zink (Tab. 4).

Tab. 3

Wiederfindung für Eisen, Kupfer und Zink in Serum. Eingesetzt wurden $4,5 \mathrm{ml}$ Serum, aufgestockt mit $0,5 \mathrm{ml}$ Lösung der angegebenen Konzentrationen

\begin{tabular}{|c|c|c|c|c|c|}
\hline \multicolumn{6}{|c|}{ Eisen } \\
\hline Probe & $\begin{array}{l}\text { erwartet } \\
{[\mu \mathrm{mol} / \mathrm{l}]}\end{array}$ & $\begin{array}{l}\text { gefunden } \\
{[\mu \mathrm{mol} / 1]}\end{array}$ & $\begin{array}{c}\text { Wieder- } \\
\text { findung } \\
{[\%]}\end{array}$ & $\mathrm{n}$ & $\mathbf{s}$ \\
\hline $\begin{array}{l}\text { Serum } \\
+50 \mu \mathrm{mol} / 1 \\
+100 \mu \mathrm{mol} / 1 \\
+150 \mu \mathrm{mol} / 1 \\
+300 \mu \mathrm{mol} / 1\end{array}$ & $\begin{array}{l}24,2 \\
29,2 \\
34,2 \\
49,2\end{array}$ & $\begin{array}{l}21,4 \\
23,6 \\
28,7 \\
34,6 \\
47,8\end{array}$ & $\begin{array}{r}97,4 \\
98,1 \\
101,2 \\
97,1\end{array}$ & $\begin{array}{l}21 \\
20 \\
23 \\
22 \\
21\end{array}$ & $\begin{array}{l}0,67 \\
0,60 \\
0,65 \\
0,81 \\
0,95\end{array}$ \\
\hline \multicolumn{6}{|c|}{ Kupfer } \\
\hline $\begin{array}{l}\text { Serum } \\
+50 \mu \mathrm{mol} / 1 \\
+100 \mu \mathrm{mol} / 1 \\
+150 \mu \mathrm{mol} / 1 \\
+300 \mu \mathrm{mol} / 1\end{array}$ & $\begin{array}{l}23,0 \\
28,0 \\
33,0 \\
48,0\end{array}$ & $\begin{array}{l}20,0 \\
22,6 \\
28,1 \\
33,4 \\
47,8\end{array}$ & $\begin{array}{r}98,3 \\
100,5 \\
101,2 \\
.99,5\end{array}$ & $\begin{array}{l}20 \\
10 \\
10 \\
10 \\
10\end{array}$ & $\begin{array}{l}0,61 \\
0,60 \\
0,69 \\
0,57 \\
0,71\end{array}$ \\
\hline \multicolumn{6}{|c|}{ Zink } \\
\hline $\begin{array}{l}\text { Serum } \\
+50 \mu \mathrm{mol} / 1 \\
+100 \mu \mathrm{mol} / 1 \\
+150 \mu \mathrm{mol} / 1 \\
+300 \mu \mathrm{mol} / 1\end{array}$ & $\begin{array}{l}16,7 \\
21,7 \\
26,7 \\
41,7\end{array}$ & $\begin{array}{l}13,0 \\
16,4 \\
22,2 \\
26,4 \\
40,8\end{array}$ & $\begin{array}{r}98,2 \\
102,3 \\
98,8 \\
97,8\end{array}$ & $\begin{array}{l}20 \\
20 \\
20 \\
20 \\
20\end{array}$ & $\begin{array}{l}0,12 \\
0,67 \\
0,19 \\
0,38 \\
0,41\end{array}$ \\
\hline
\end{tabular}

Tab. 4

Wiederfindung unterhalb von $10 \mu \mathrm{mol} / \mathrm{l}$. Gefundener Wert: $\overline{\mathbf{x}} \pm \mathrm{s}$, Probenzahl: $n=6$ für Eisen, $n=3$ für Kupfer und Zink

\begin{tabular}{ccccccc}
\hline $\begin{array}{c}\text { Probe } \\
{[\mu \mathrm{mol} / 1]}\end{array}$ & \multicolumn{2}{c}{ Eisen } & \multicolumn{2}{c}{ Kupfer } & \multicolumn{2}{c}{ Zink } \\
& $\overline{\mathrm{x}}^{[\mu \mathrm{mol} / 1]}$ & $\pm \mathrm{s}$ & $\overline{\mathrm{x}}^{[\mu \mathrm{mol} / 1]}$ & $\pm \mathrm{s}$ & $\overline{\mathrm{x}}^{[\mu \mathrm{mol} / 1]}$ & $\pm \mathrm{s}$ \\
\hline 2 & 1,9 & 0,42 & 1,9 & 0,08 & 2,0 & 0,03 \\
4 & 4,1 & 0,48 & & & & \\
5 & 5,6 & 0,61 & 4,6 & 0,08 & 5,1 & 0,08 \\
\hline
\end{tabular}

Aus Tabelle 4 wird ersichtlich, daß die Methode auch bei niedrigen Konzentrationen ausreichend genaue Werte liefert, um z. B. den klinisch bedeutsamen Mangelzustand im Eisenhaushalt zu erkennen.

Im zweiten Teil der Wiederfindungsversuche sollte geprüft werden, wie verläßlich bei Serienmessungen Konzentrationen direkt am DCR-1 ablesbar waren. Kurvenausgleiche waren nicht erforderlich, da Linearität für alle drei Eichgeraden bis $80 \mu \mathrm{mol} / \mathrm{l}$ bestand. Für Eisen und Kupfer wurden die Konzentrationen 10 und $60 \mu \mathrm{mol} / 1$ in das DCR-1 eingegeben und die Wiederfindung für 20 bzw. $40 \mu \mathrm{mol} / \mathrm{l}$ geprüft. Für Zink erfolgte die Eingabe von 10 und $40 \mu \mathrm{mol} / \mathrm{l}$, die Wiederfindung für 15 und $20 \mu \mathrm{mol} / \mathrm{l}$. Bei einer geringen Anzahl $(n<10)$ von Proben ergab dieses Vorgehen ausreichend genaue Ergebnisse. Die Wiederfindung für Eisen, Kupfer und Zink lag nur dann um $100 \%$, wenn nach mehrfachen Versuchen die eingegebenen Eichpunkte etwa konstant blieben. Zudem zeigte sich, daß bei Messung einer größeren Probenzahl ( $n>10)$ das allmähliche Abweichen des Nullpunktes auftrat. Der Nachteil ist offensichtlich. Es müssen zeitraubende Korrekturen vorgenommen werden, wobei die alleinige Nullpunktskorrektur nicht ausreicht. Der Verbrauch an Eichlösungen ist dann beträchtlich.

Aufgrund dieser Beobachtungen wurden die Konzentrationen der Probandenseren mit Hilfe eines Eichpunktes nach der oben angegebenen Formel ermittelt, da sich das Eingabeverfahren für unser Vorhaben als unzweckmäßig erwies. Zwischenzcitlich auch jetzt notwendige Korrekturen ließen sich jedoch schneller durchführen.

\section{Präzision in Serie}

Die Präzision in Serie zeigt Tabelle 2 mit dem Ergebnis der Serienbestimmungen zur Prüfung der Linearität der Eichkurven.

\section{Präzision von Tag qu Tag}

Es wurde ein handelsübliches Kontrollserum (Seronorm) verwandt, das Eisen, Kupfer und, wie wir fanden, auch Zink enthielt. Angaben über die Präzision von Tag zu Tag enthält Tabelle 5.

\section{Vergleich verschiedener Meßmethoden}

Vergleichsbestimmungen haben wir für Kupfer und Eisen durchgeführt, nicht jedoch für Zink, da wir dieses Element bisher mit einer anderen Methode nicht bestimmten.

Tab. 5

Präzision von Tag zu Tag an n Tagen

\begin{tabular}{lcccc}
\hline & $\mathrm{n}$ & $\begin{array}{c}\overline{\mathbf{x}} \\
{[\mu \mathrm{miol} / 1]}\end{array}$ & $\begin{array}{c} \pm \mathrm{s} \\
{[\mu \mathrm{mol} / 1]}\end{array}$ & $\begin{array}{c}\text { VK } \\
{[\%]}\end{array}$ \\
\hline Eisen & 21 & 22,3 & 0,88 & 3,9 \\
Kupfer & 21 & 11,4 & 0,42 & 3,6 \\
Zink & 21 & 10,1 & 0,36 & 3,6
\end{tabular}




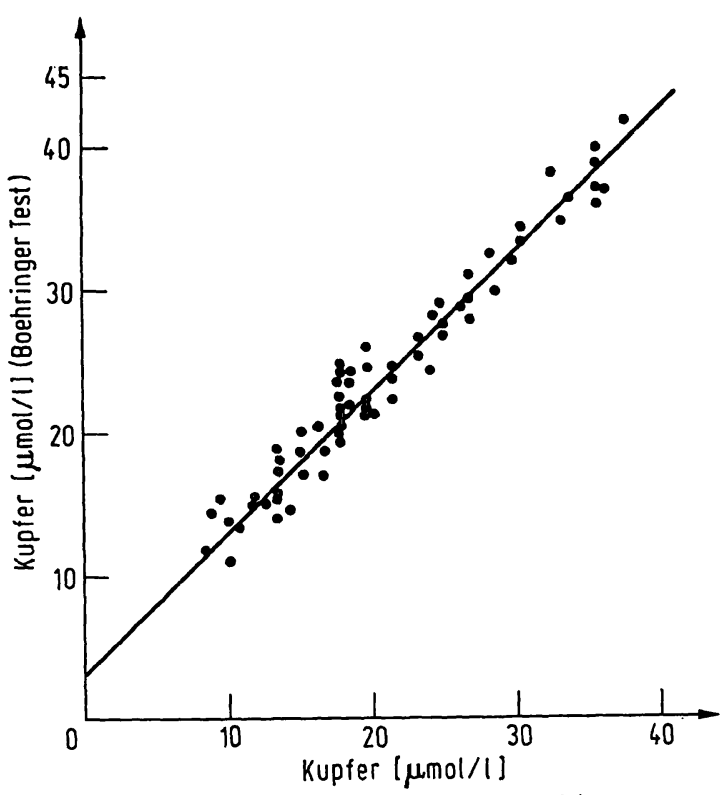

(Atom- Absorptions-Spektrometrie)

Abb. 2

Korrelationsdiagramm für die Serumkupferwerte, die mit dem Boehringer-Testbesteck (Ordinate) und dem Atom-Absorptions-Spektrometer (Abszisse) bestimmt wurden. $N=76, y=3,20+0,9997 x$, bestimmt wurden. $N=76, y$
$\mathrm{~S}_{\mathbf{y}} \cdot \mathrm{x}= \pm 1,65$ und $\mathrm{r}=0,976$

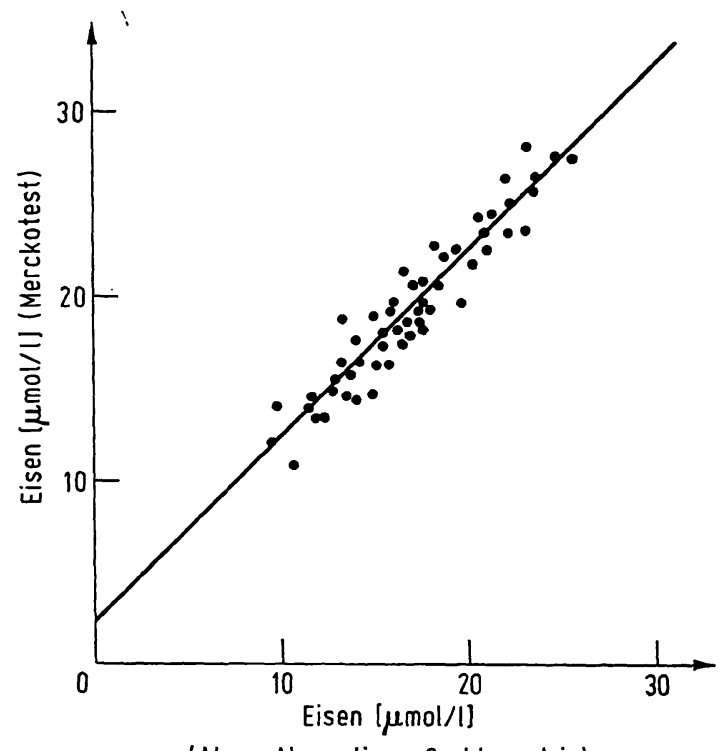

(Atom-Absorptions-Spektrometrie)

Abb. 3

Korrelationsdiagramm für die Plasmaeisenwerte, die mit dem MerckTestbesteck (Ordinate) und dem Atom-Absorptions-Spektrometer (Abszisse) ermittelt wurden. $\mathrm{N}=55, \mathrm{y}=2,28+1,0107 \mathrm{x}$,
$\mathrm{S}_{\mathbf{y}} \cdot \mathrm{x}= \pm 1,31$ und $\mathrm{r}=0,9516$

Bei 76 routinemäßig gewonnenen Patientenseren wurden sowohl nach der beschriebenen Methode wie auch mit dem Boehringer-Testbesteck nach $\mathrm{Z}_{\mathrm{AK}}$ (7) die Kupferkonzentrationen ermittelt. Der Korrelationskoeffizient betrug $\mathrm{r}=0,976$, die Regressionsgerade $\mathrm{y}=$ $3,2+0,9997 x$ und die Streuung $s_{y \cdot x}= \pm 1,65$ wie Abbildung 2 zeigt.

Die Eisenkonzentrationen im Plasma von 55 Probanden wurden nach unserer oben beschriebenen Methode und mit dem Merck-Testbesteck nach Schwartz (8) bestimmt. Die Hämoglobinwerte haben wir gleichzeitig nach der von RICHTERICH (9) beschriebenen Methode
Tab. 6

Angabe von $\bar{x} \pm s$ der Eisenwerte im Plasma sowie der Hb-Konzentrationen vor und nach Hämolyse

\begin{tabular}{lcccc}
\hline & $\begin{array}{c}\text { Proben- } \\
\text { zahl }\end{array}$ & $\begin{array}{c}\text { Eisen im Plasma } \\
{[\mu \text { mol/l] }} \\
\text { Atom-Ab- Merck-Test- } \\
\text { borption }\end{array}$ & $\begin{array}{c}\text { Hämoglobin im } \\
\text { Plasma } \\
{[\mathrm{mg} / 1]}\end{array}$ \\
\hline Vor Hämolyse & 55 & $\begin{array}{c}16,9 \pm 3,9 \\
\text { Nach Hämolyse }\end{array}$ & $19,4 \pm 4,2$ & $356,6 \pm 87,2$ \\
\hline
\end{tabular}

(Normalwert: $50-400 \mathrm{mg} / \mathrm{l}$ ) gemessen. Weiterhin ermittelten wir die Eisen- und $\mathrm{Hb}$-Werte im Plasma einer zweiten teilhämolysierten Blutprobe dieser Probanden. Es zeigte sich eine positive Korrelation der Eisenwertepaare von der Hämolyse, wobei die mit der AtomAbsorption bestimmten Konzentrationen niedriger lagen (Abb. 3). Der Korrelationskoeffizient war $\mathrm{r}=0,951$, die Regressionsgerade $\mathrm{y}=2,28+1,0107 \mathrm{x}$ und die Streuung $s_{y} \cdot x= \pm 1,31$. Nach der Teilhämolyse erhöhten sich die Hb-Konzentrationen auf etwa das dreibis vierfache des Ausgangswertes, und es kam zu einem erheblichen Anstieg der mit der Atom-Absorption gemessenen Eisenwerte. Auch die Eisenwerte, die mit dem Merck-Testbesteck bestimmt wurden, ethöhten sich um 3-4 $\mu \mathrm{mol} / \mathrm{l}$ (Tab. 6).

Normbereiche für Eisen im Plasma, Kupfer und Zink im Serum

Die graphische Darstellung in einer Häufigkeitskurve ließ bei allen drei Elementen eine rechtsschiefe Verteilung erkennen. Aus der statistischen Berechnung ging hervor, daß für Eisen im Plasma, Kupfer und Zink im Serum eine angenähert log-normale Verteilung vorliegen dürfte und somit eine Angabe von Mittelwert und 2s-Bereich nicht zulässig ist. Dieser Verteilungstyp konnte für Eisen im Plasma wie auch Kupfer und Zink im Serum im Wahrscheinlichkeitsnetz mit logarithmischer Abszisse bestätigt werden. Wir haben deshalb die 95\%-Normbereiche sowie den Median nach SACHS (10) ermittelt und mit Normalwerten anderer Autoren in Tabelle 7 zusammengestellt.

\section{Diskussion}

Die Atom-Absorptions-Spektrometrie bietet zweifelsohne zahlreiche Vorteile und Arbeitserleichterungen $(3,19-22)$ gegenüber anderen Methoden und ihre Möglichkeiten dürften im Hinblick auf die große $\mathrm{Zahl}$ an bestimmbaren Elementen noch nicht voll ausgeschöpft sein.

Die Anwendung der Atom-Absorptions-Spektrometrie in der klinisch-chemischen Diagnostik zur Bestimmung von Eisen im Plasma, Kupfer und Zink im Serum läßt aber einige Probleme zutage treten.

Wir entschieden uns für die direkte Messung der Proben. Dieses Vorgehen erspart mehrere Arbeitsschritte, die anderen zeitaufwendigen Probenvorbereitungen zugrunde liegen und häufig Fehlerquellen beinhalten. Es ist jedoch darauf hinzuweisen, daß die 
Tab. 7

Zusammenstellung von Normalwerten für Eisen, Kupfer und Zink im Serum. Lediglich die Zinkwerte von HALSTED (14), GEISLER (17) und unsere eigenen Eisenwerte wurden im Plasma ermittelt. Für die eigenen Werte wird der Median und der $95 \%-N o r m b e r e i c h$ angegeben

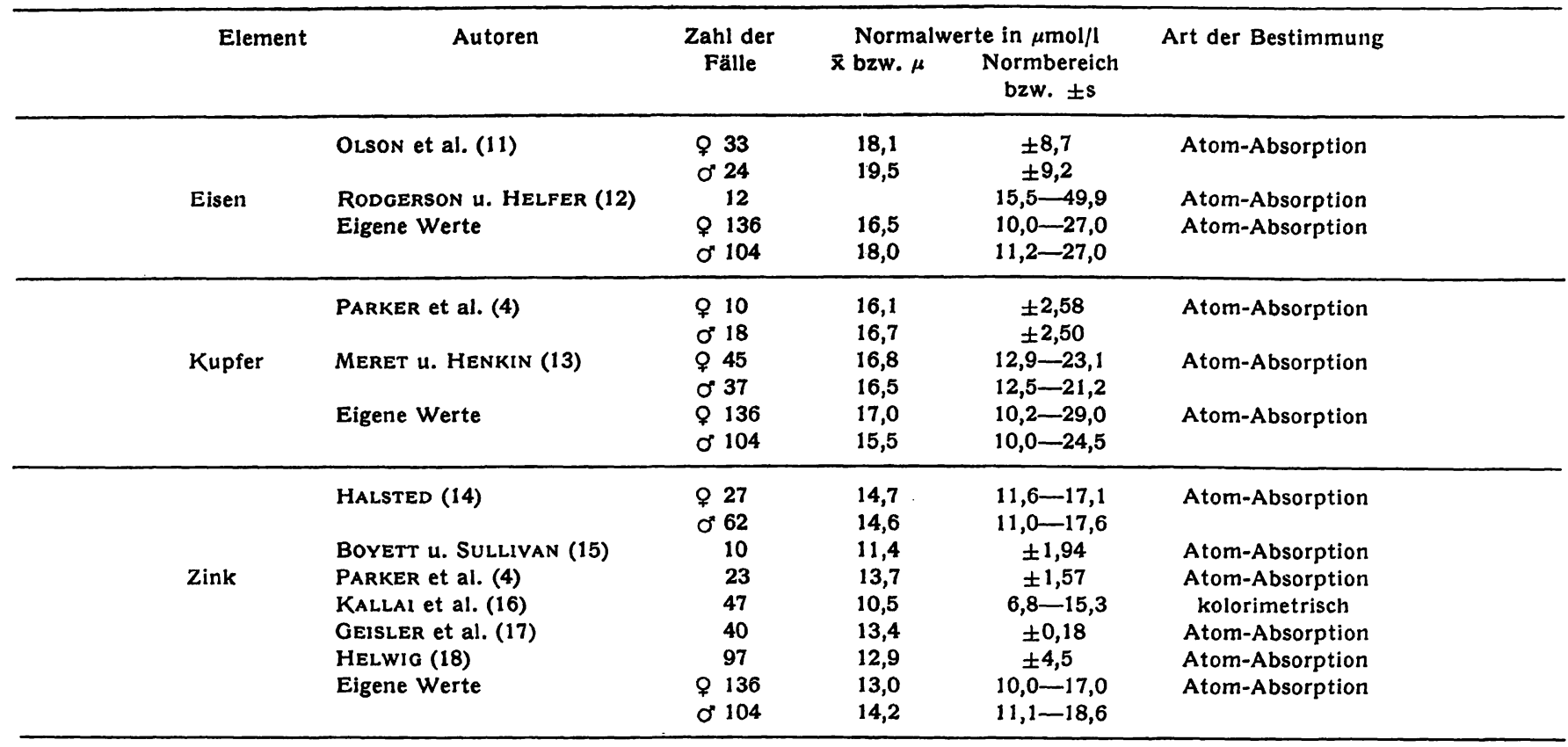

einfache Verdünnung der Proben mit schon niedrigen Konzentrationen an Eisen, Kupfer und Zink das Signal-Rauschverhältnis zwangsläufig beeinträchtigt. Ein anderes Problem ergibt sich bei einer größeren Probenzahl aus der allmählich eintretenden Nullpunktsabweichung des Gerätes. Diese ist einerseits auf den elektrischen Geräteteil, andererseits auf zunehmende Verunreinigung des Brennerkopfes zurückzuführen. Geringe Korrekturen sind also unvermeidbar.

Zur Verwendung von neo-Plasmagel bei der Herstellung der Eichlösungen sind zwei Gründe zu erwähnen: Erstens sollte eine ähnliche Viskosität von verdünntem Serum und Eichlösungen erreicht werden, was für die Zerstäubung bedeutsam ist. Zweitens liegt Natrium in physiologischer Konzentration im neoPlasmagel vor. Die Bedeutung der Anwesenheit von Natrium bei den Absorptionsvorgängen der Eisenund Kupferbestimmung wurde neuestens wieder hervorgehoben (6). Natrium soll durch Verschiebung des Ionisationsgleichgewichtes zu einer Erhöhung der Kupfer- bzw. Erniedrigung der Eisenwerte führen.

Die Angaben über den Einfluß von Natrium auf die Zinkwerte sind widersprüchlich $(2,23)$.

Die Linearität der Eichkurven für Eisen, Kupfer und Zink reichte über die Konzentrationen hinaus, welche die bei Kranken zu erwartenden Werte einschließen. Die Prüfung der Richtigkeit brachte eine Wiederfindungsrate bei allen drei Elementen zwischen 97,4 und 102,3\%. Weiterhin zeichnet sich die Methode durch gute Präzisionen in der Serie wie auch von Tag zu Tag aus.

Werden Eisenbestimmungen mit der Atom-Absorptions-Spektrometrie durchgeführt, dann kann vorerst nur die Verwendung von Plasma empfohlen werden, was durch die positive Korrelation für Wertepaare im Plasma in Abbildung 3 unterstrichen wird. Unsere kolorimetrisch ermittelten, gering höheren Werte für Eisen im Plasma entsprechen einer Angabe von ZETTNER et al. (24), der mit der Atom-Absorptions-Spektrometrie gleichfalls niedrigere Werte als mit einem Farbtest erhielt. Für Eisenwertepaare aus routinemäßig gewonnenem Serum dagegen ließ sich keine Korrelation feststellen. Ursächlich in Betracht dafür kam die Hämolyse, die bei nicht korrekter Blutabnahme und beim Gerinnungsvorgang in mehr oder minder starker Form auftrat. Das freigesetzte Hämoglobineisen wurde also neben dem transferringebundenen Eisen bei der Atom-Absorptions-Spektrometrie miterfaßt. Daraus resultierten die zu hohen Werte. Normalerweise werden ähnlich hohe hämolysebedingte Hämoglobinkonzentrationen wie in Tabelle 6 angegeben nicht erreicht, doch kann die Erhöhung der Serumeisenwerte auch bei noch nicht sichtbarer Hämolyse deutlich sein. Gerinnung und Blutabnahme sind entscheidende Einflüsse bei dieser Eisenbestimmung.

Eine mögliche Fehlerquelle ist bei jenen Seren zu berücksichtigen, die hämolytisch sind und zusätzlich andere Chromogene in höherer Konzentration enthalten, wodurch die Hämolyse überdeckt wird und sich unerwartet hohe Eisenwerte einschleichen können.

Die Eisenbestimmung mit dem Merck-Testbesteck dagegen ergab nach Teilhämolyse nur gering höhere Eisenwerte. Auch SCHADE et al. (25) weisen auf die geringe Beeinflußbarkeit der photometrischen Eisenbestimmung durch Hämolyse hin.

Vielleicht bringt in Zukunft die Bestimmung von Eisen im Vollblut mit dem neuen Verfahren der Graphitrohrküvette mehr, da hierbei die Hämolyse unberücksichtigt bleiben dürfte. 
Die von uns ermittelten Mediane und Normbereiche sind mit neueren Literaturangaben ( $\mathrm{Tab}$. 7) vergleichbar, sofern man sie im Hinblick auf das gleiche Bestimmungsprinzip auswählt. Zahlreichen Normalwertangaben für Eisen, Kupfer und Zink liegt ein kolorimetrisches Meßverfahren zugrunde. Diese Werte bieten kein einheitliches Bild, wie aus einer Tabelle "Normalwerte der Plasmaeisenkonzentration" in 1. c. (9) mit teilweise sehr weiten Normbereichen hervorgeht. Auch für Kupfer (26) und Zink (27) im Serum werden in einer Tabelle Normalwerte mehrerer Autoren zitiert, die ebenfalls deutliche Abweichungen voneinander zeigen. Es sei erwähnt, daß viele dieser Autoren ihre Ergebnisse mit kleinen Probandenzahlen gewonnen haben und keine Angaben zur Verteilung machen. Wir fanden für die drei Elemente eine angenähert log-normale Verteilung im Serum bzw. Plasma.

Der Zeitpunkt der Blutabnahme ist bei Eisen-, Kupferund Zinkbestimmungen von Bedeutung, weil für Eisen stärkere Schwankungen des Plasmaspiegels innerhalb eines Tages (Abnahme bis zum Abend 20-30\%) und auch von Woche zu Woche auftreten $(28-30)$. Eine Tagesthythmik mit einem durchschnittlichen Abfall von 8.00 bis $17.00 \mathrm{Uhr}$ von $4,58 \mu \mathrm{mol} / 1$ wird auch für Zink mitgeteilt (31). Für Kupfer finden sich weniger tageszeitliche Schwankungen, jedoch von Tag zu Tag bis zu $4,7 \mu \mathrm{mol} / 1$ (32).

Über Änderungen der Serumwerte für Zink nach Nahrungszufuhr, insbesondere Kohlenhydraten, berichten Davies et al. (33). Der Anstieg des Serumzinks nach Aufnahme von Kohlenhydraten gilt als Folge der Ausschüttung verschiedener zinkhaltiger Enzyme. Eisen und Kupfer zeigen nach Nahrungszufuhr keine signifikante Änderung des Serumspiegels (34).

Abschließend sei erwähnt, daß Eisen- und Kupferwerte bei Frauen durch Ovulationshemmer beeinflußt werden können (35).

\section{Literatur}

1. Sprague, S. \& Slavin, W. (1965), At. Absorption Newslett. 4, 228-233. - 2. Hackley, B. M., Smith, J. C. \& Halsted, J. A. (1968), Clin. Ghem. 14, 1-5. - 3. Ringhardtz, J. \& Welz, B. (1968), Z. Analyt. Chem. 243, 190-198. - 4. PArker, M. M., Humoller, F. L. \& Mahler, D. J. (1967), Clin. Chem. 13, 40-48. - 5. Fuwa, K., Pulido, P., McKay, R. \& Vallee, B. L. (1964), Anal. Chem. 36, 2407-2411. - 6. Schмid, W. (1968), Z. Analyt. Chem. 243, 198-208. - 7. ZAK, B. (1958), Clin. Chim. Acta 3, 328-334. - 8. Schwartz, D. E. (1957), Bulletin der Schweiz. Vereinigung für klinische Chemie Nr. 1. - 9. Richterich, R. (1971), Klinische Chemie, 3. Aufl., 387-388 u. 247-251, Verlag S. Karger, Basel. - 10. SACHS, H. (1968), Statistische Auswertungsmethoden, 1. Aufl., 66-109, Verlag Springer, Berlin. - 11. Orson, A. D. \& Hamlin, W. B. (1969), Clin. Chem. 15, 438-444. - 12. Rodgerson, D. O. \& Helfer, R. E. (1966), Clin. Chem. 12, 338-348. - 13. Meret, S. \& Henkin, R. I. (1971), Clin. Chem. 17, 369-373. - 14. Halsted, J. \& S Sirth jr., J. C. (1970), Lancet I, 322-324. - 15. BOYETT, J. D. \& Sullivan, J. F. (1970), Metabolism 19, 148-157. 16. Kallai, L., Keler-Bacoka, Mira, Marinkovic, M., Knezevic, S., Stojanovski, Andrina \& Kosutic, Z. (1968), Schweiz. Med. Wochenschr. 98, 1007-1009. - 17. GeIsler, Ch., StaCher, A., Stöckl, W. \& Werser, M. (1972), Wien. Klin. Wochenschr. 84, 171-175. - 18. Helwig, H. L., Unveröffentlichte Mitteilung zit. nach: KahN, A. M., Helwig, H. L., Redeker, A. G. \&
Reynolds, T. B. (1965), Amer. J. Clin. Pathol. 44, 426-435. 19. Allan, J. E. (1961), Spectrochim. Acta 17, 459-466. 20. HerrmanN, R. (1965), diese Z. 3, 178-186. - 21. Wiltis, J. B. (1960), Spectrochim. Acta 16, 259-272. - 22. Paschen, K. \& Spiekermani, P. G. (1970), Deut. Med. Wochenschr. 51, 2577-2581. - 23. Prasad, A. S., Oberleas, D. \& Halsted, J. A. (1965), J. Lab. Clin. Med. 66, 508-516. - 24. Zetrner, A., Sylvia, L. C. \& Capacho-Delgado, L. (1966), Amer. J. Clin. Pathol. 45, 533-540. - 25. Schade, A. L., Oyama, J., Reinhart, R. W. \& Mriler, J. R. (1954), Proc. Soc. Exp. Biol. Med. 87, 443-448. - 26. Dawson, J. B., Elels, D. J. \& Newţon-John, H. (1966), Clin. Chim. Acta 21, 33-42. - 27. Dawson, J. B. (1969), Clin. Chim. Acta 26, 465-475. - 28. Bowre, E. J. W., Tauxe, W. H., SJoberg, W. E. \& Yamaguchi, M. Y. (1963), Amer. J. Clin. Pathol. 40, 491-494. - 29. Hoyer, K. (1944), Acta. Med. Scand. 119, 562-576. - 30. Thedering jr., F. (1949), Klin. Wochenschr. 27, 496-501. - 31. Hellwege, H. (1970), Klin. Wochenschr. 48, 1063-1064. - 32. RrCE, E. W. (1962), Amer. J. Med. Sci. 243, 593-597, - 33. Davres, I. J., MusA, M. \& Dormandy, L. (1968), J. Clin. Pathol. 21, 359-363. 34. Moore, C. V. \& Dubach, R. (1962), in Mineral Metabolism (Comar, C. L. \& Bronner, F., Hrsg.) 1. Aufl., Bd. 2, Teil B, 287-348, Academic Press, New York. - 35. Wilbert, L., Hillimer, T., Hunstein, W., Reisert, P., Kaboth, U. \& CreutzFELD, W. (1969), Deut. Med. Wochenschr. 16, 844-849.

\footnotetext{
Dr. G. Heinemann

Institut für Klinische Chemie und Klinische Biochemie Klinikum Steglitz der FU Berlin

1 Berlin 45

Hindenburgdamm 30
} 